REVISTA PROYECCIONES N $N^{\circ} 12: 113-114$

Diciembre 1986 - ISSN 0716-0917

Jornada Matemáticas, Agosto 1986.

\title{
COMPORTAMIENTO ASINTOTICO DE UN SISTEMA NO LINEAL DE ECUACIONES DIFERENCIALES PARCIALES
}

\section{Dr. ELIAS TUMA*}

En este trabajo se desarrolla un principio de comparación para las ecuaciones de Fitz Hugh - Nagumo en conjuntos acotados.

$$
\begin{aligned}
& u_{t}=u_{x x}+f(u)-v, x \in \Omega_{\underline{C}} \mathbb{R}, t>0 \\
& v_{t}=\sigma u-\gamma v
\end{aligned}
$$

donde $f(u)=u(1-u)(u-a), 0<a<1, \sigma \geqslant 0, \gamma \geqslant 0$ y $\Omega$ es un conjunto acotado en $\mathbb{R}$. Más aún estudiamos el comportamiento asintótico de las soluciones de (1) en el caso de que éste admite tres estados estacionarios constantes. Consideramos condiciones de frontera e iniciales para (1) de la forma :

$$
B[u]=\alpha(x) \frac{\partial u(x, t)}{\partial \vec{n}}+\beta(x) u(x, t)=b(x, t), t>0, x \in \partial \Omega
$$

\footnotetext{
* Universidad Técnica Federico Santa María. Valparaiso - Chile.
} 
(3) $u(x, 0)=u_{0}(x), v(x, 0)=v_{0}(x), x \in \Omega$, donde $\vec{n}$ es el vector unitario normal a la frontera $\partial \Omega, \alpha \geqslant 0, \beta \geqslant 0 \operatorname{con} \alpha(x)+\beta(x)>0, x \varepsilon \partial \Omega ; u_{0}(x)$, $v_{0}(x) \in C^{1}(\Omega)$ y $u_{0}(x)$ satisface $(2) \operatorname{con} \alpha(x), \beta(x), b(x, t)$ en $C^{1}\left(\partial \Omega \times\left(\mathbb{R}^{+} \times\{0\}\right)\right)$.

El problema (1) ha sido extensamente estudiado por varios matemáticos (ej: J. Rauch, J. Smoller. Qualitative Theory of the Fitz Hugh Nagumo equations. Advances in Math. (27), 12-44, (1978)). Usando Rectángulos Contractbles, Métodos de Energía, Perturbaciones, etc. Aquí nues tra atención es establecer una nueva técnica que permite estudiar el comportamiento cualitativo de las soluciones de (1) - (2) - (3). 